Paidéia, 2006, 16(34), 215-223

\title{
A NATUREZA DA REPRESENTAÇÃO DE CENAS VISUAIS: EVIDÊNCIAS BASEA- DAS NO EFEITO DE TAREFAS INTERVENIENTES SOBRE A MEMÓRIA PARA DISTÂNCIAS ENTRE OBJETOS ${ }^{1}$
}

\author{
Náira Cristina Alcântara Verceze \\ Susi Lippi Marques \\ Universidade Federal de São Carlos \\ Cesar Alexis Galera ${ }^{2}$ \\ Universidade de São Paulo - FFCLRP
}

\begin{abstract}
Resumo: Três tarefas intervenientes (verbal, visual e aritmética), realizadas durante estudo de uma cena, foram utilizadas com o objetivo de explicitar a natureza da representação memorizada das distâncias entre os objetos da cena. $\mathrm{O}$ efeito delas sobre a representação das distâncias foi estimado por expoentes da função potência baseados em julgamentos da distância relembrada entre pares de objetos e reprodução em desenho da disposição espacial dos objetos da cena. Os resultados mostram que expoentes obtidos não diferem da unidade. Por outro lado, os estimados pelas coordenadas x e y com base nos desenhos são menores. Tanto os expoentes obtidos nos julgamentos de magnitude de distância aos pares como aqueles a partir do eixo y do desenho foram afetados pela realização da tarefa aritmética durante o tempo de estudo, mas não pela realização da tarefa visual. Estes resultados sugerem que componentes visuais e espaciais contribuem com pesos diferenciados para a qualidade da representação.
\end{abstract}

Palavras-chave: memória visoespacial; tarefas intervenientes; estimação de magnitude; desenho.

\section{THE NATURE OF THE REPRESENTATION OF SHORT-TERM MEMORIZED SCENES: THE EFFECT OF INTERVENING TASKS ON THE MEMORY FOR THE DISTANCES BETWEEN OBJECTS}

\begin{abstract}
Three intervening tasks (verbal, visual and arithmetical) performed by participants during the study of a scene were used to explicit the nature of the memorized representations of the distances between scene objects. The effect of the intervening task on the distances representation was determined through the exponent of the power function estimated from the judgments of distances based on estimation and the distances between objects in scene. The results showed that the exponents obtained by magnitude estimation do not differ from unit. On the contrary, the exponents obtained on the basis of the $\mathrm{x}$ and $\mathrm{y}$ coordinates of the drawing have lower values. The exponents obtained with judgments of magnitude and from the y axe of the drawing were affected by the arithmetical task performed during the study time, but not by visual one. These results suggest that visual and spatial components contribute with differentiated weights for the representation quality.
\end{abstract}

Key words: visuo-spatial memory; intervening tasks; magnitude estimation; drawing.

\section{Introdução}

A natureza da representação da informação visual tem ocupado um lugar de destaque na Psicologia dos últimos 40 anos. As evidências sugerem que as representações mentais refletem processos aná-

\footnotetext{
${ }^{1}$ Recebido em 16/08/05 e aceito para publicação em 27/06/06.

${ }^{2}$ Endereço para correspondência: Cesar Alexis Galera, Departamento de Psicologia e Educação da Faculdade de Filosofia Ciências e Letras de Ribeirão Preto - USP, Av. Bandeirantes 3900, CEP 14040901, Ribeirão Preto - SP, E-mail: algalera@usp.br
}

logos àqueles envolvidos na percepção de objetos do ambiente (Shepard \& Metzler, 1971). As evidências também sugerem que a forma como se julgam objetos com base no que se lembra deles é análoga à forma como se julga objetos presentes diante dos olhos (Moyer, 1973). O método de julgamento de magnitude proposto por Stevens (1975), assim como o uso da função potência, tem sido muito útil no estudo da relação funcional entre a magnitude psicológica e 
física dos estímulos. A Lei de Stevens tem sido utilizada para avaliar vários parâmetros, dentre os quais: sonoridade, peso, brilhância, comprimento, área, volume e distância visual (Stevens, 1975). Essa é descrita através da seguinte função: $R=K E^{n}$, em que $\mathrm{R}$ é a magnitude psicológica, $\mathrm{K}$ é uma constante que depende da unidade de medida, E representa a magnitude física do estímulo e N é o expoente da função, que simboliza o índice de sensibilidade para cada modalidade perceptiva, sendo o parâmetro mais importante da função (Stevens, 1975).

Vários estudos têm aplicado a lei de Stevens em situações em que os julgamentos se baseiam em estímulos memorizados e têm mostrado, por exemplo, que para as modalidades visuais, os expoentes obtidos no julgamento de área e distância feitos com base na memória são geralmente menores do que os obtidos nas condições perceptivas (Algom, Wolf \& Bergman, 1985; Radvansky, Carlson-Radvansky \& Irwin, 1995; Marques, 1996). Outros estudos apontam que o processo de julgamento exigido nos experimentos de percepção é muito semelhante aos exigidos nos baseados em imagens mentais (Hubbard \& Stoeckig, 1992; Kosslyn, Ball \& Reisser, 1978; Kerst \& Howard, 1978, Baird \& Harder, 2000).

Duas hipóteses foram levantadas para explicar as diferenças entre os expoentes obtidos com estímulos presentes e a partir de representações memorizadas. O Modelo da Tendência Central de Julgamentos considera que o rebaixamento dos expoentes de estimativas relembradas vem do aumento da incerteza sentida pelo observador na condição de julgamento de memória (Marques \& Da Silva, 1998). A incerteza seria o resultado da perda de informações nos traços de memória, o que levaria o participante a efetuar suas estimativas tendendo ao centro da escala de julgamentos, estando, portanto, as estimativas de memória envolvidas com o grau de confiança com que o observador faz seus julgamentos (Radvansky, Carlson-Radvansky \& Irwin, 1995).

O Modelo Re-perceptual defende que o expoente de magnitude relembrada será igual ao quadrado do de magnitude perceptiva. Este modelo sugere a intervenção de duas funções de potência entre a magnitude física real e a estimativa da dimensão através da memória. A primeira é tipicamente perceptiva e a segunda é introduzida pelo processo de relembrar (Kerst \& Howard, 1978; Marques \& Da Silva, 1998). Neste sentido, pode-se supor que as representações utilizadas nos julgamentos de memória são análogas às usadas nos julgamentos perceptivos e, que ambas são representações visoespaciais. De fato, existem evidências de que muitas estruturas do córtex relacionadas ao processamento primário da percepção são as mesmas envolvidas nas imagens mentais (Farah, 1985, 1989), mas existem diferenças a respeito do que isso significa em termos da existência das imagens mentais análogas (Kosslyn, Ganis \& Thompson, 2003).

Investigou-se a natureza da representação memorizada da disposição de objetos em cenas reais através do efeito de tarefas intervenientes de subtração e de imaginação visual, sobre as estimativas de distâncias entre os objetos da cena. Existem evidências de que a tarefa de aritmética afeta a capacidade da memória visual de curto prazo, embora ainda não seja claro se isto ocorre porque os recursos atencionais necessários ao armazenamento visual são drenados pela tarefa de subtração (Philips \& Cristie, 1977a, 1977b), ou porque a tarefa de subtração interfere diretamente no armazenamento da localização dos estímulos (Lee \& Kang; 2002; Galera \& Fuhs, 2003). Por outro lado, a tarefa de imaginação visual afeta o desempenho das que dependem do armazenamento de informação visual, mas não interfere no desempenho das verbais (Logie, Zucco \& Baddeley, 1990; Logie, 1995). Os resultados têm mostrado que a tarefa de subtração, realizada no intervalo de retenção, diminui o expoente das estimativas de distância entre os objetos da cena enquanto que a realização de uma tarefa de imaginação visual aumenta esse expoente (Marques \& Galera, 2002; Marques, Galera \& Eik, 2000).

Além disso, de acordo com os resultados, a representação das distâncias entre os objetos também é afetada pela familiaridade que os participantes possam ter com os objetos. Cenas com objetos familiares (embalagens de produtos comerciais tais como lata de leite condensado, extrato de tomate) foram representadas com "maior precisão" do que cenas com objetos não familiares (cilindros coloridos de madeira), sugerindo que as representações mentais, 
além da informação espacial dos objetos, mantém também, informação não espacial (Marques \& Galera, 2002).

No presente estudo investigou-se a natureza da representação memorizada de uma cena através do efeito das tarefas intervenientes realizadas durante o estudo da cena. A suposição subjacente é que o sistema cognitivo dispõe de recursos específicos e limitados para codificar e armazenar informação no curto prazo. Dessa forma, tarefas intervenientes que utilizem os mesmos recursos necessários à codificação da cena terão efeitos mais acentuados sobre a recordação dela do que tarefas intervenientes que utilizem recursos irrelevantes para a tarefa em questão.

\section{Método}

\section{Participantes}

Participaram do estudo 18 estudantes universitários com idades variando entre 18 a 25 anos. Os colaboradores eram ingênuos quanto ao propósito do estudo e não tiveram contato prévio com as cenas ${ }^{1}$.

\section{Material}

Foram utilizadas quatro cenas montadas com quatro tipos de objetos. Cada uma continha sete objetos distribuídos sobre uma mesa com $1,2 \mathrm{~m}$ x 1,2m e $0,76 \mathrm{~m}$ de altura, forrada com tecido xadrez vermelho e branco. Cada cena foi gerada a partir de uma configuração espacial básica (Figura 1) apresentada em quatro orientações. Desta forma, as posições ocupadas pelos objetos nelas guardavam as mesmas relações espaciais. A mesa foi montada em uma sala com paredes pintadas de preto, com apenas um ponto de luz central iluminando-a. Os participantes observaram a configuração através de uma janela aberta apenas no intervalo dedicado ao estudo da cena) com o queixo apoiado sobre um suporte a $0,40 \mathrm{~m}$ da mesa e a uma altura de $1,20 \mathrm{~m}$.

Na montagem da cena foram utilizados produtos alimentícios familiares (lata de pêssego, lata de leite condensado, lata de guaraná, fermento em pó, geléia e extrato de tomate), produtos de beleza ou higiene pessoal (batom, desodorante, talco, perfume, $\underline{\text { shampoo, }}$, condicionador e gel), garrafas de bebida

\footnotetext{
${ }^{1}$ O projeto foi submetido e aprovado pelo Comitê de Ética da
} UFSCar. pequenas (isotônico, refrigerante, suco, água, cerveja long neck, garrafa de vinho e mini-pet), e objetos cilíndricos com $12 \mathrm{~cm}$ de altura e $5 \mathrm{~cm}$ de diâmetro apresentados em amarelo, rosa, roxo, preto, verde, azul e laranja. Uma quinta configuração, formada também por sete objetos (sabonete, shampoo, cilindro marrom, gelatina, ervilha, pasta de dente, garrafa de iogurte) foi utilizada para a realização de treino do participante antes do início do experimento.

Os participantes registraram as estimativas (julgamentos de distância entre pares de estímulos) em cadernos que apresentavam o nome de doze pares de objetos em uma ordem aleatória e, identificaram a localização espacial dos estímulos utilizando uma folha de papel milimetrado medindo $12 \times 12 \mathrm{~cm}$.

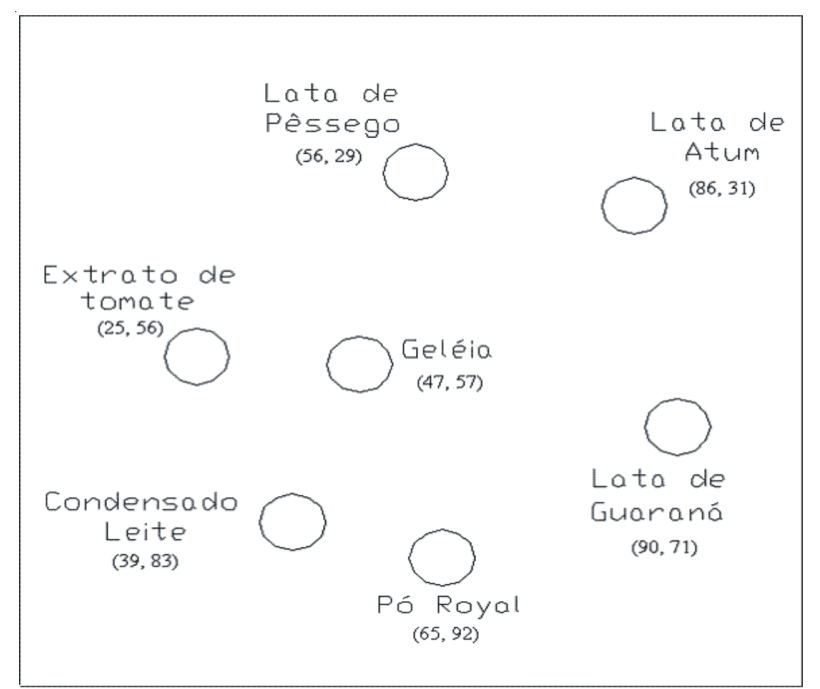

Figura 1. Exemplos de objetos utilizados para compor as cenas utilizadas. Entre parênteses as coordenadas $\mathrm{x}$ e y em centímetros, considerando o canto superior esquerdo como o ponto $(0,0)$.

\section{Procedimento}

Depois de receberem instruções para a conduta do experimento e após terem assinado o termo de consentimento livre e esclarecido, os participantes passaram individualmente por um treino sobre as tarefas que realizariam ao longo do experimento. Basicamente a tarefa era estudar uma configuração com sete objetos durante o intervalo máximo de dois minutos. Imediatamente depois deveria realizar estimativas de distância entre os objetos da cena ou desenhar sua distribuição espacial. Foram utilizadas três tarefas intervenientes que o participante deveria exe- 
cutar durante o estudo da cena até o momento em que iniciava sua tarefa de estimação de magnitude (com apresentação do estímulo padrão) ou de produção do desenho. Na tarefa de imaginação visual, baseada na tarefa utilizada por Logie, Zucco e Baddeley (1990), foi pedido a eles que visualizassem uma matriz com três colunas e cinco linhas. Quando informavam ao experimentador que tinham uma imagem clara da matriz, eles ouviam uma seqüência de instruções para preencher, ou deixar em branco, cada uma das células da matriz imaginada. Ao final dessa seqüência de instruções, um dos dígitos de 0 a 9 deveria estar "visível" na matriz, e o participante deveria dizer qual era esse dígito. A literatura revela que esta tarefa interfere no armazenamento da informação visual (Logie, 1995, 1996). A segunda tarefa interveniente, tarefa aritmética, consistiu em contar em voz alta, de

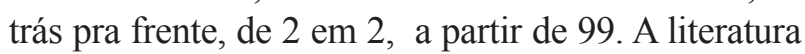
sugere que tarefas aritméticas simples como esta interferem com a capacidade da memória espacial, possivelmente por utilizarem um código visuo-espacial analógico (Dehaene 1992; Lee \& Kang, 2002; Smyth, Pearson \& Pendleton; 1988; Smyth \& Pelky, 1992). A terceira tarefa interveniente, de supressão verbal, consistiu simplesmente em repetir a expressão blá, blá, blá, ou seja, fala irrelevante, durante o estudo da cena. Esta tarefa interfere na recitação sub-vocal, prejudicando a capacidade da memória verbal (Baddeley, 1986). Numa quarta situação experimental os participantes não foram submetidos à tarefa interveniente. Todos passaram por todas as tarefas e configurações de objetos. Os participantes receberam instruções para realizar o julgamento de magnitude entre os estímulos requeridos nos cadernos de respostas e uma folha de papel milimetrado, proporcional ao tamanho da mesa com os objetos, onde deveriam reproduzir a posição e nomear os objetos observados. Metade dos participantes realizou inicialmente julgamento de magnitude e, em seguida, o desenho; e a outra metade realizou inicialmente o desenho e depois o julgamento. A ordem pela qual passaram pelas diferentes tarefas, assim como pelas diferentes configurações de objetos foi aleatorizada.

\section{Resultados}

As estimativas de magnitude entre as distâncias dos objetos e o desenho da cena foram utilizadas para estimar os expoentes da função potência. Para a obtenção dos expoentes a partir dos desenhos considerou-se a distância entre as coordenadas $\mathrm{x}$ e y de cada objeto na configuração e as coordenadas $\mathrm{x}$ e y da posição ocupada pelo objeto na reprodução gráfica da cena realizada pelos sujeitos. Dessa forma, foram obtidos dois expoentes para cada desenho, um baseado na distância na coordenada $\mathrm{x}$ e outro na coordenada y. Os resultados de dois participantes na produção de desenho foram desconsiderados para a análise dada a sua qualidade gráfica insatisfatória.

Inicialmente os expoentes de 16 participantes cujas médias são apresentadas na Figura 2, foram submetidos a uma análise de variância para medidas repetidas tendo as três formas utilizadas para estimar os expoentes (julgamento, estimativa em x e estimativa em y) e os quatro tipos de tarefas intervenientes (visual, verbal, aritmética e sem tarefa) como fatores experimentais. Esta análise mostrou que os expoentes variam em função da técnica utilizada para obtê-los $\left(\mathrm{F}_{(2,30)}=3,38, \mathrm{p}=0,047\right)$. Uma análise de comparação aos pares (NewmannKeuls, $\mathrm{p}<0,05)$ confirmou que o expoente médio obtido com o julgamento de magnitude $(1,00)$ é maior do que o expoente médio obtido a partir da coordenada y $(0,73)$, mas não do que aquele obtido com base na coordenada $\mathrm{x}(0,80)$.

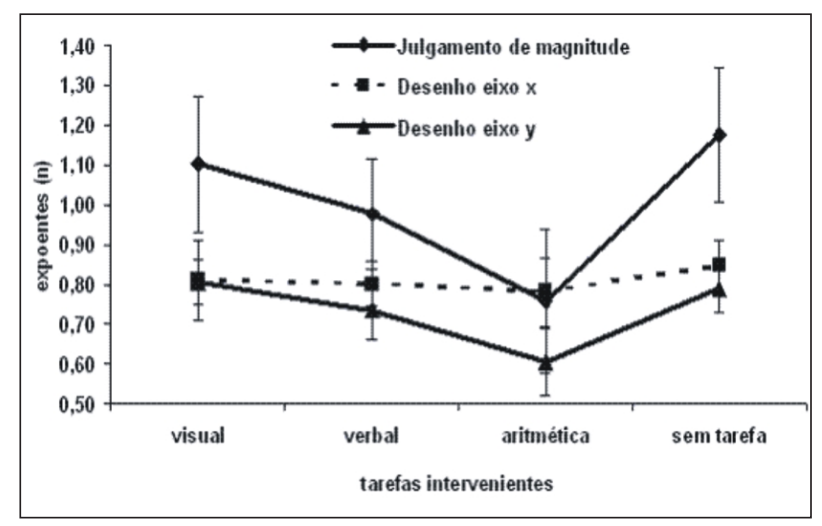

Figura 2: Expoente médio da função de potência para os diferentes tipos de tarefas intervenientes para julgamento de magnitude e desenho da cena nos eixos $\mathrm{x}$ e $\mathrm{y}$.

As tarefas intervenientes têm efeitos significativos sobre os expoentes obtidos através de julgamentos de magnitude $\left(\mathrm{F}_{(3,45)}=2,87, \mathrm{p}<0,05\right)$. Neste caso, os expoentes obtidos com a realização simultâ- 
nea das tarefas verbal, visual e sem tarefa interveniente são típicos de tarefas de estimação de distâncias nas quais os expoentes variam entre $0,91 \mathrm{e}$ 1,00 (Stevens \& Guirao, 1963; Teghtsoonian, 1965; Teghotsoonian \& Teghtsoonian, 1978; Cruz, 1991). Entretanto, o expoente obtido com a tarefa aritmética é menor $(0,76)$ do que esses valores típicos. O mesmo padrão acontece para os expoentes obtidos a partir das distâncias no eixo y dos desenhos $\left(\mathrm{F}_{(3,45)}=\right.$ $3,99, \mathrm{p}<0,01)$; neste caso o expoente obtido com a tarefa aritmética foi menor $(0,49)$ do que os expoentes obtidos em todas as outras tarefas (média igual a 0,76). Os expoentes obtidos a partir das distâncias no eixo x sugerem que a representação dessas distâncias não é afetada por qualquer uma das tarefas intervenientes empregadas $(\mathrm{F}<1)$.

Posto que na literatura vários estudos de estimação de distância apontam para um expoente variando de $0,91-1,00$, pode-se apreender que os valores do expoente de estimação de magnitude apresentados neste estudo $(0,96)$ não devem ser considerados diferentes do valor típico de 1,0 encontrado na literatura $(\mathrm{t}(\mathrm{gl}=15)=0,273, \mathrm{p}=0,39)$. Contudo, os expoentes obtidos a partir dos desenhos, com média de 0,75 , são significantemente menores $(\mathrm{t}(\mathrm{gl}=15)=$ $6,7, \mathrm{p}<0,001)$. Supõe-se então que esta diferença poderia estar associada à forma de julgamento imposta pelas técnicas utilizadas (julgamento de magnitude e produção de magnitude através do desenho).

\section{Discussão}

Neste estudo procurou-se investigar as características da representação memorizada das distâncias entre objetos de uma cena. Três tarefas intervenientes foram utilizadas com o objetivo de explicitar a natureza dessas representações. A qualidade das representações foi estimada através do expoente da função potência baseado em julgamentos da distância relembrada e da reprodução em desenho das distâncias entre os objetos da cena. Na tarefa de estimação de magnitude de distância, obtevese um valor equivalente ao valor típico encontrado na literatura, tanto para estimativas feitas a partir da cena percebida como para julgamentos feitos a partir de representações relembradas (Algom \& Goshen, 1994). Os resultados mostraram que o expoente obtido a partir dos julgamentos de magnitude foi maior do que aqueles obtidos a partir da reprodução gráfica da cena, e que a tarefa interveniente aritmética tende a produzir uma redução tanto dos expoentes obtidos a partir de julgamentos de magnitude como nos expoentes estimados a partir do deslocamento no eixo y das reproduções gráficas. Resultados semelhantes foram encontrados por Marques, Galera \& Eik (2000), numa pesquisa na qual a tarefa interveniente foi aplicada durante os sete minutos no intervalo de retenção entre o estudo e a reprodução da cena. A realização da tarefa aritmética no intervalo de retenção também provocou uma diminuição significativa dos expoentes obtidos através dos desenhos, mas não teve um efeito significativo sobre os expoentes obtidos através da estimação de magnitude no estudo de memória a longo prazo.

Resultados encontrados na literatura têm sugerido que a tarefa aritmética utiliza recursos comuns à memória visuo-espacial. Phillips e Christie (1977a, b) apontaram que a recordação de seqüências de padrões visuais sem nome é fortemente afetada pela realização simultânea de uma tarefa aritmética. Quando os recursos necessários ao armazenamento da cena são drenados pela tarefa aritmética a informação memorizada é degradada. Resultados semelhantes foram obtidos por Lee e Kang (2002) em que os sujeitos realizaram tarefas aritméticas de subtração e de multiplicação combinadas com tarefas de memória fonológica e visoespacial. A realização de uma tarefa verbal prejudicou a tarefa de multiplicação, mas não afetou a tarefa aritmética, no caso subtração. Por outro lado, manter na memória a posição na qual um padrão visual foi apresentado prejudicou a realização da tarefa aritmética, mas não teve efeito significante sobre o desempenho na tarefa de multiplicação. De acordo com os autores esses resultados indicam que a tarefa de subtração utiliza recursos relacionados ao sistema visuo-espacial.

Evidentemente a relação entre os recursos utilizados na codificação e armazenamento da informação visoespacial e a realização de tarefas aritméticas depende da natureza das tarefas aritméticas investigadas. Noel, Désert, Aubrun e Seron (2001) mostraram o envolvimento do circuito fonológico na realização de uma tarefa aritmética no caso em que o participante soma números com um dígito. Os estudos de Logie, Gilhooly e Wynn (1994) mostraram que 


\section{Náira Cristina Alcântara Verceze}

o executivo central também pode ser envolvido na soma de números com dois dígitos. Técnicas de tomografia por emissão de pósitrons mostram que tarefas de cálculo aritmético simples $(16+6$, por exemplo) envolvem áreas normalmente associadas ao circuito fonológico do modelo de memória operacional (giro angular do parietal esquerdo), mas também envolvem áreas associadas ao comportamento motor e ao controle atencional (ínsula e tálamo médio) (Cowell, Egan, Code, Harasty \& Watson, 2000). No estudo de Zago e cols. (2001) a execução de cálculos complexos ( $32 \times 24$, por exemplo) também é acompanhada de um aumento da ativação na área parieto-frontal esquerda e no giro temporal inferior bilateral, áreas associadas ao processamento da informação visuo-espacial. De acordo com Dehaene (1997), diferentes áreas cerebrais são requisitadas em diferentes momentos do cálculo. Inicialmente, os símbolos aritméticos são transformados num código fonológico, mas o processamento desses símbolos durante o cálculo propriamente dito envolveria a manipulação da informação tanto em termos verbais quanto em termos visoespaciais analógicos.

O cálculo aritmético parece utilizar os mesmos sistemas de armazenamento em comum com a informação visoespacial e exige recursos atencionais que também parecem envolvidos na codificação desse tipo de informação. Numa revisão do modelo de memória operacional, Miyake Friedman, Rettinger, Shah e Hegarty (2001) consideram que existe uma relação íntima entre a memória operacional visuoespacial, o executivo central e habilidades espaciais. De fato, a relação parece tão íntima que chega a comprometer a possibilidade de separar o funcionamento do executivo central do funcionamento da memória visuo-espacial de curto prazo (Duff \& Logie, 1999). Embora a distinção entre esses componentes do sistema de memória operacional tenha ainda que ser feita em um nível mais central da análise, a memória visuo-espacial fornece as melhores evidências para subdivisões adicionais da memória operacional, sugerindo, pelo menos, que o processo espacial e o visual podem se basear em pools separados de recursos. Evidências nesse sentido são fornecidas pelos padrões de interferência obtidos com o paradigma da tarefa dupla, que mostraram que a manutenção da informação espacial na memória operacional é preju- dicada pela realização de uma tarefa espacial simultânea, mas não por uma tarefa visual concorrente, enquanto que a manutenção da informação visual pode ser prejudicada por uma tarefa visual simultânea mas não por uma tarefa espacial simultânea (Logie, 1995).

A ausência do efeito da tarefa interveniente de imaginação visual é bastante interessante, pois sugere que a codificação e a representação memorizada da cena e a tarefa de imaginação visual não concorrem pelos mesmos recursos. Antes que se possa interpretar este resultado é necessário considerar que os obtidos neste estudo podem ter sido produzidos, não pela ação direta da tarefa interveniente, mas pela estratégia adotada pelo participante. A realização da tarefa interveniente, concomitante com o estudo da cena, pode ter sido feita em termos de um "time sharing". Nesse caso haveria um custo em coordenar as duas atividades. De acordo com Carlson, Wenger e Sullivan (1993), em muitas tarefas, os indivíduos devem coordenar a informação mantida na memória operacional (memória a curto prazo) com a informação disponível no ambiente para apresentar uma seqüência apropriada de ação. Esta coordenação é freqüentemente requerida porque tarefas cognitivas envolvem a combinação da informação na memória de curto prazo com a informação sobre aspectos do ambiente. Neste caso, se se admitir que houve uma estratégia de coordenação na forma de "time sharing", dever-se-á explicar também o motivo pelo qual a coordenação é mais fácil para a tarefa de imaginação visual e mais difícil para a aritmética. Dessa forma, se se considerar que os resultados em termos de recursos necessários à realização da tarefa interveniente e à codificação da cena poder-se-á supor que o componente visual é mais estável e menos sujeito à interferência das tarefas intervenientes do que o espacial. No caso do presente estudo, devido às características dos estímulos, isto é, condição de estimulação familiar, a visualização de objetos com os quais se tinha uma história prévia de contato, pode ter influenciado e facilitado o acesso a tais representações internas. Assim sendo, o desempenho dos participantes pode ter sido favorecido com este tipo de estímulo. Também é possível que a coordenação da tarefa de imaginação visual e o estudo da cena seja mais fácil do que a coordenação da realização da tarefa aritmética e do estudo da cena. Estas 
questões deverão ser investigadas oportunamente e os dados oriundos deste estudo poderão servir de base para a construção e testagem de hipóteses. Outro aspecto importante a ser analisado futuramente será a questão de nomeação dos estímulos, tendo em vista as colocações de Olson e Bialystok (1983) a partir das quais sugerem que as propriedades espaciais dos estímulos podem ser utilizadas para o reconhecimento de objetos (através da forma); para lembrar localizações espaciais, através de seu posicionamento ou por pontos de referência, entre outros julgamentos. Ainda, fatores referentes a agrupamentos de elementos baseando-se em sua proximidade e similaridade serão investigados e discutidos (Tversky , 2000; Galera \& Fuhs, 2003).

Pôde-se apreender que os dados desta pesquisa corroboram a noção de que a representação memorizada dos objetos de uma cena é feita em termos visuo-espaciais, e sugere que os componentes visuais e espaciais contribuem com pesos diferenciados para a qualidade da representação. Neste sentido, considerando que a tarefa aritmética utilizou recursos visuo-espaciais necessários à codificação de uma representação mental estável da cena estudada, deve-se supor que os dados obtidos nesse estudo dariam maior suporte ao Modelo Re-perceptual, proposto por Kerst e Howard (1978) para explicar o rebaixamento dos expoentes estimados a partir de representações memorizadas.

De maneira geral, deve-se ainda atentar para a diferença dos expoentes obtidos através das técnicas de julgamento adotadas. $\mathrm{O}$ expoente obtido através da estimação de magnitude foi igual a 1,0 e o expoente para distâncias desenhadas foi menor $(0,81$ para o eixo x e 0,73 para o eixo y). No desenho, as distâncias entre os objetos são mais comprimidas do que no julgamento de magnitude. Esta diferença, em primeira instância, pode refletir uma compressão determinada pela escala na qual é realizada a estimativa e pela técnica de produção de magnitude, isto é, representar graficamente a configuração. A diferença no valor do expoente obtido na coordenada y em relação à x pode ser elucidada pelo efeito da profundidade nas representações visuais. Um estudo em campo aberto realizado por Loomis, Da Silva, Fujita e Fukusima (1992) demonstrou que os participantes, diante de uma mesma distância, percebem diferente- mente profundidades e comprimentos, sendo as primeiras consideradas inferiores às segundas. Isto se justificaria pela maior dificuldade do homem em realizar julgamentos em profundidade e, no caso deste estudo, em reproduzir a configuração espacial dentro destes parâmetros.

Aprofundando a análise na direção da produção do desenho, percebe-se que esta técnica mostra-se complexa e exigente na medida em que o participante além de ter que representar a cena memorizada numa dimensão diferente da estudada tem que efetivamente apontar a localização e a identidade do estímulo. Na situação da estimação de magnitude o julgamento é um tanto mais facilitado, pois as estimativas das distâncias são solicitadas aos pares e apresentadas nominalmente ao participante. Assim, a configuração é avaliada em seqüências específicas e julgada via um padrão de comparação. Uma vez que este padrão de julgamento para estimativas de magnitude e produção de desenho têm sido sistematicamente observado em estudos anteriores (Marques, Galera \& Eik, 2000; Marques \& Galera, 2002), podese supor que as técnicas utilizadas podem acessar diferentes propriedades das representações memorizadas. Neste sentido parece que o expoente obtido no estudo da representação mental da cena é dependente da técnica adotada na estimativa de distância entre pares de estímulos e desenho.

Finalizando, dentro de suas possibilidades informativas, a presente investigação evidenciou que a representação memorizada dos objetos de uma cena é feita em termos visoespaciais, e sugere que os componentes visuais e espaciais contribuem diferentemente na qualidade da representação. Ainda, foram apontadas a viabilidade e aplicabilidade da produção do desenho da cena como um instrumento importante para o estudo da representação mental, oferecendo-se dessa forma, uma contribuição metodológica ao estudo da natureza da representação de cenas visuais.

\section{Referências}

Algom, D. \& Goshen, E. (1994). Remembered and perceived size as a function of familiarity. Perceptual and Motor Skills, v.78, n.33 1035-1040.

Algom, D., Wolf, Y. \& Bergman, B. (1985). Integration of stimulus dimensions in perception and memory: composition rules and psychophysical 
relations. Journal of Experimental Psychology: General, v.114, n.44, 451-71.

Baddeley, A. (1986). Working Memory. NY: Oxford University Press.

Baird, J. C. \& Harder, K. A. (2000). The psychophysics of imagery. Perception \& Psychophysics, v. 62, n.11, 113-126.

Carlson, R. A., Wenger, J. L. \& Sullivan, M. A. (1993). Coordinating information from perception and working memory. Journal of Experimental Psychology: Human Perception and Performance, v.19, n.3, 531-549.

Cowell, S. F., Egan, G. F., Code, C., Harasty, J., Watson, J. D. (2000). The functional neuroanatomy of simple calculation and number repetition: A parametric PET activation study. Neuroimage, v.12, n. 5, 565-73.

Cruz, M. A. C. Q. M. (1991). Canais visuais separados para percepção de comprimento, distância, área e volume: Enfoque da psicofisica experimental. Dissertação de Mestrado, FFCLRP, Universidade de São Paulo, Ribeirão Preto.

Dehaene, S. (1992). Varieties of numerical abilities. Cognition, 44, 1-42.

Dehaene, S. (1997). La bosse des maths. Paris: Editions Odile Jacob.

Duff, S. C. \& Logie, R. H. (1999). Storage and processing in visuo-spatial working memory. Scandinavian Journal of Psychology, 40, 251-259.

Farah, M. (1985). Psychophysical evidence for a shared representational medium for mental images and percepts. Journal of Experimental Psychology: General, 114, 91-103.

Farah, M. (1989).The neural basis of mental imagery. Trends in Neurosciences, v.12, n.10, 395399.

Galera, C. \& Fuhs, C. (2003). A capacidade da memória visuo-espacial a curto prazo: o efeito de tarefas de supressão verbal e aritmética. Psicologia: Reflexão e Crítica, v.16, n.2, 337 - 348.

Hubbard, T. L. \& Stoeckig, K. (1992). The representation of pitch in musical images. In D.
Reisberg (Ed.), Auditory Imagery (pp. 199-235). Hillsdale, N. J.: Lawrence Erlbaun Associates.

Kerst, S. M. \& Howard, J. H. (1978). Memory psychophysics for visual area and length. Memory and Cognition, v.6, n.3, 237-335.

Kosslyn, S. M., Ball, T. M. \& Reisser, B. J. (1978). Visual images preserve metric of spatial information: evidence from studies of image scanning. Journal of Experimental Psychology: Human Perception and Performance, 4, 47-60.

Kosslyn, S. M., Ganis, G. \& Thompson, W. L. (2003). Mental imagery: Against the nihilistic hypothesis. Trends-in-Cognitive-Sciences, 7, 109111

Lee, K-M. \& Kang, S. Y. (2002). Arithmetic operation and working memory: differential suppression in dual tasks. Cognition, v.83, n.3, B63B68.

Logie, R. H. (1995). Visuo-spatial working memory. UK: Lawrence Erlbaum Associates.

Logie, R.H. (1996). The seven ages of working memory. In J.T.E. Richardson (Ed.), Working memory in human cognition (pp. 31-65). Oxford: Oxford Press.

Logie, R. H., Gilhooly, K. J. \& Wynn, V. (1994). Counting on working memory in arithmetic problem solving. Memory \& Cognition, v.22, n.44, 395-410.

Logie, R. H., Zucco, G. M. \& Baddeley, A. (1990). Interference with visual short-term memory. Acta Psychologica, v.75, n.1, 55-74.

Loomis, J. M., Da Silva, J. A., Fujita, N. \& Fukusima, S. S. (1992). Visual space perception and visually directed action. Journal of Experimental Psychology: Human Perception and Performance, v.18, n.4, 906-921.

Marques, S. L. (1996). Funções psicofísica perceptivas e mnemônicas de área e volume familiares e não familiares sob instruções aparente e objetiva. Tese de Doutorado, FFCLRP, Universidade de São Paulo, Ribeirão Preto.

Marques, S. L. \& Da Silva, J. A . (1998). Psicofísica da memória: efeitos de pistas visuais e de 
métodos psicofísicos sobre estimativas perceptivas e mnemônicas. Arquivos Brasileiros de Psicologia, v. 50, n. 1/2, 108-123.

Marques, S. L. \& Galera, C. (2002). The effect of arithmetic and visuo-spatial tasks on memory for items and their spatial location. In J. A. Da Silva, E.H. Matsushima \& N. P. Ribeiro-Filho, (Eds.), Proceedings of the Eighteenth Annual Meeting of the International Society for Psychophysics, 18 (pp.464-469). Rio de Janeiro: The International Society for Psychophysics.

Marques, S. L., Galera, C. \& Eik, G. (2000). Efeito de tarefas intervenientes verbais e visuoespacias sobre estimativas mnemônicas em diferentes procedimentos psicofísicos. Arquivos Brasileiros de Psicologia, v.52, n.1, 26-34.

Miyake, A., Friedman, N. P., Rettinger, D. A., Shah, P. \& Hegarty, M. (2001). How are visuospatial working memory, executive functioning, and spatial abilities related? A latent-variable analysis. Journal of Experimental Psychology: General, 130, 621640

Moyer, R. (1973). Comparing objects in memory: Evidence suggesting an internal psychophysics. Perception \& Psychophysics, 13, 180-184.

Noel, M. P., Desert, M., Aubrum, A. \& Seron, X. (2001). Involvement of short-term memory in complex mental calculation. Memory \& Cognition, v.29, n.11, 34-42.

Olson, D. R. \& Bialystok, E. (1983). Spatial cognition: the structure and development of mental representations of spatial relations. New Jersey: Lawrence Erlbaum Associates.

Phillips, W. A. \& Christie, D. F. M. (1977a). Components of visual memory. Quarterly Journal of Experimental Psychology, 29, 117-133.

Phillips, W. A. \& Christie, D. F. M. (1977b). Interference with visualization. Quarterly Journal of Experimental Psychology, 29, 637-650.

Pylyshyn, Z. (2003). Return of the mental image: Are there really pictures in the brain? Trendsin-Cognitive-Sciences, v. 7, n.3, 113-118.
Radvansky, G. A., Carlson-Radvansky, L. A. \& Irwin, D.E. (1995). Uncertainty in estimating distances from memory. Memory \& Cognition, v.23, n.5, 596-606.

Shepard, R.N. \& Metzler, J. (1971). Mental rotation of three dimensional objects. Science, 171, 701-703.

Smyth, M.M., Pearson, N.A., \& Pendleton, L.R. (1988). Movement and working memory: Pattern and positions in space. Quarterly Journal of Experimental Psychology, 40A, 497-514.

Smyth, M. M. \& Pelky, P. (1992). Short-term retention of spatial information. British Journal of Psychology, v.83, n.3, 359-374.

Stevens, S. S. (1975). Psychophysics: Introduction to its perceptual, neural and social prospects. New York: Wiley.

Stevens, S. S. \& Guirao, M. (1963). Subjective scaling of length and area and the matching of length to loudness and brightness. Journal of Experimental Psychology, 66, 177-186.

Teghtsoonian, M. (1965). The judgment size. American Journal of Psychology, 78, 392-402.

Teghtsoonian, R. \& Teghtsoonian, M. (1978). Range and regression effects in magnitude scaling. Perception \& Psychophysics, 24, 305-314.

Tversky, B. (2000). Remembering spaces. In E. Tulving \& F. I. M. Craik, (Eds.), Handbook of Memory. (pp.363-378). New York: Oxford University Press.

Zago, L., Pesenti, M., Mellet, E., Crivello, F., Mazoyer, B. \& Tzourio-Mazoyer, N. (2001). Neural correlates of simple and complex mental calculation. NeuroImage, 13, 314-327.

Apoio: $\mathrm{CNPq}$ 